\title{
L'âge: une contre-indication au traitement psychique?
}

François Villa

L'âge est-il une contre-indication au traitement psychique? L'auteur montre que, derrière la question de l'âge, c'est la dimension économique qu'il faut prendre en considération.

Mots clés: Économique (dimension), pulsion, traitement psychique, vieillissement 
J'ai ici l'impression que je devrais avoir honte de toutes ces discussions pesantes, ce qu'elles disent étant connu depuis longtemps et allant de soi. Effectivement nous nous sommes toujours comporté comme si nous savions cela, à cette réserve près que dans nos représentations théoriques nous avons négligé la plupart du temps de tenir compte du point de vue économique dans la même mesure que des points de vue dynamique et topique. Mon excuse c'est donc que je remets en mémoire cette négligence.

(Freud, 1937, p. 241-242).

Cette surprenante remarque surgit dans ce texte tardif où Freud s'interroge sur les fins de la cure en les questionnant radicalement. L'ombilic de la cure, ce point où l'interprétation n'est plus de mise, est ici appelé “roc de la castration”: véritable roc d'origine où le psychique rencontre le fait biologique comme le lieu indépassable de son origine. C'est, sous l'angle de cette négligence de l'économique, que nous aborderons les quelques remarques ${ }^{1}$ faites par Freud sur l’âge comme contre-indication du “traitement de l’âme”.

Ce n’est pas tant le nombre d’années qui est désigné, que la dimension du temps comme favorisant le renforcement de la dimension économique: "Et tout se passe comme si, véritablement, la victoire était la plupart du temps du côté des bataillons les plus fort” (Freud, 1937, p. 255). Du début à la fin de l’œuvre, sera maintenue cette position. Que la contre-indication concerne, dans les textes de 1904 et de 1905, “les personnes ayant atteint ou

1. À notre connaissance, quatre textes évoquent la question de la contre-indication de l’âge : 1) "De la méthode psychanalytique de Freud” (Freud, 1904, p. 17-18); 2) “De la psychothérapie” (Freud, 1905), 3) “Extrait de l'histoire d’une névrose infantile. (L'homme aux loups)” (Freud, 1914[1918], p. 415), 4) “L’analyse avec fin et l'analyse sans fin” (Freud, 1937, p. 237, 241, 257). 
dépassé la cinquantaine” (c’est l'âge de Freud à cette période) et dans celui de 1937 (l'auteur a alors 81 ans) “des personnes très âgées” peut prêter à sourire. La limite d’âge ne serait-elle déterminée que par l’âge de Freud pour qui le traitement analytique serait, en quelque sorte, toujours contre-indiqué?

Tant dans "De la méthode psychanalytique de Freud" que dans “De la psychothérapie”, un des éléments imposant des limites au traitement est le point de butée de la constitution (Freud, 1915-1917, 340) qui ne saurait être dépassée: elle se traduit par des résistances insurmontables. Parmi les autres éléments, certains ne sont pas tant déterminés qu'accentués par la prise d’âge. Le matériel psychique à perlaborer, en raison de sa masse, ne peut plus être terrassé, on n’en vient plus à bout (bewältigen ${ }^{2}$ ). Cela "prolonge la durée de la cure qui s'étend à l'infini”, "le temps nécessaire à la production, au rétablissement devient trop long”. La capacité de "faire rétrograder” (rückgängig zu machen) le processus psychique commence à s'affaiblir, à être paralysée (beginnt zu erlahmen). Les vieilles gens ne disposent plus de la plasticité des processus psychiques, c'est pour cela qu'elles ne sont plus éducables et qu'elles sont peu influençables.

\section{La dimension métapsychologique}

Tous ces termes, qui justifient la contre-indication de la psychanalyse, renvoient à la dimension métapsychologique. En avançant qu'à partir d'un certain âge, le temps de cure nécessaire au "rétablissement" deviendrait trop long, Freud indique que le travail psychique s’inscrit dans une durée, qu’il nécessite du temps pour s'accomplir. Il est le travail de toute une vie par lequel le vivant tente de donner figure humaine à son existence. A un certain moment, entre le temps nécessaire au processus et la durée de vie encore probable, l'écart est tel, qu’il n’est plus temps d'entreprendre une telle démarche, car le travail psychique à accomplir peut paraître démesuré. La quantité de matériel refoulé, dont il faudrait se rendre maître pour ne pas être débordé par les forces maintenues jusque-là réprimées, est telle, que, dans le temps probable qu'il reste à vivre, la perlaboration - traduction dynamique et topique d'un changement des investissements topiques - est peu envisageable.

Dans le temps d'une vie, il est des moments où quelque chose arrive qui, pour être supporté, exige que nous puissions, pour partie au moins, en maîtriser l'ex-

2. Traduit étonnamment en français par “étudier à fond”, ce verbe dessine un tout autre paysage, bien moins intellectualisé, plus dynamique et plus violent, cf. Laplanche, 1989, p. 149-150. 
citation qui en résulte, la lier à des représentations qui en permettent la figuration. Il existe des instants opportuns à l'élaboration, l'exigeant même, sous peine de se voir livrer à l'infini ressassement et, lorsqu'ils ne sont pas saisis, un trop de choses en souffrance se constituent dont nulle figuration n'est advenue. La capacité d'élaborer ce qui arriva, jadis, se voit compromise, car elle n'est ni infinie, ni a-temporelle. Ce qui aurait dû être un tant soit peu déjà pensé est tel que cela excède ce que la pensée pourrait maintenant en élaborer: l'excessif de la tâche est alors menace de débordement et de “décompensation” pour l'appareil psychique.

Ce n'est donc pas l'âge en tant que nombre d'années qui constitue une contre-indication, mais ce qu'il en est de la qualité de processus qui s'accomplissent dans la durée? L’idée d’âge, depuis la triple dimension de l'abord métapsychologique, est saisie par ce processus que N. Abraham a nommé anasémique. Une désignification des mots les plus communs se produit et ils redeviennent alors le lieu où résonne l'étendue de la Psyché.

Les mots, qui accompagnent la survenue de l'âge dans les textes de Freud, détachent le processus du vieillissement de la stricte chronologie et l'inscrivent dans la durée d'un ensemble de processus physiologiques et psychiques. Ceuxci ne sont pas spécifiques d'un âge donné, ils sont en jeu à tout âge et tout au long d'une vie; ce qui, dans la durée, en est modifié ce sont les rythmes, les rapports d'intensités et les équilibres précaires qui en résultent. C’est la capacité de déplacement des quantités et de leur transformation en qualité qui, avec le temps, rencontre une limite, diminue et peut être paralysée.

\section{Le caractère d'irréversibilité des processus et état d'entropie}

Dans “L'homme aux loups", la position se précise nettement: les raisons de la contre-indication ne sont pas mécaniquement liées au nombre d'années en soi, mais à quelque chose dont l'action, présente tout au long de la vie, se manifeste de plus en plus nettement au cours du temps. Voici comment Freud situe son observation: "nous ne savons qu'une chose, c'est que la qualité de mobilité des investissements psychiques recule de façon frappante avec l'âge" (Freud, 1914[1918]a, p. 112-113), il apparaît ainsi "une limite d'âge habituelle” (une moyenne) - qui cette fois-ci, notons-le, n'est plus chiffrée. Et si, il est de nouveau indiqué que cette observation "nous a fourni une des indications pour les limites de l'influence exercée par la psychanalyse”, le ton n'est plus aussi péremptoire que dans les deux textes précédents; car, "il y a des personnes chez qui cette plasticité psychique continue à exister au-delà de la limite d'âge habituelle et d'autres chez qui elle se perd précocement”. 


\section{ARTIG O S}

Pour expliquer ce plus ou moins de plasticité, qui se traduit par "la mobilité aisée ou la fluidité difficile des investissements d'énergie, libidinaux et autres”, Freud postule l'existence d'un “caractère particulier” de la pulsion: la viscosité où il reconnaît quelque chose de non-divisible comme "un nombre premier”. C'est sur la puissance de ce caractère que bute le pouvoir de l'analyse. Selon les formes où il se manifeste, il peut, “dans des conditions apparemment égales”, permettre des modifications chez certains et pas chez d'autres. Freud recourt alors au "concept d'une entropie" dont il faudrait "tenir compte dans la conversion des processus psychiques”. Cette tendance à l'entropie empêche la rétrogradation (Rückbildung) du processus et produit de l'irréversibilité. On ne peut pas rendre non-advenu (Ungeschehenmachen ${ }^{3}$ ), quelle que soit la tentation d'annulation rétroactive, ce qui est déjà advenu (geschehen). "L’analyse avec fin et l'analyse sans fin” tente de penser ce point de non-divisibilité, de non-réversibilité en l'homme sur lequel vient buter le pouvoir de la cure psychanalytique et c'est à ce propos qu'est avancé, de manière surprenante, que, la plupart du temps, le point de vue économique a été négligé dans les représentations théoriques.

\section{Ni pessimisme, ni optimisme... rencontre d'une limite}

Lire "L'analyse avec fin et l'analyse sans fin”, à partir de notre interrogation sur l'âge, est une expérience d'inquiétante étrangeté. Se dégage, en effet, l'impression que le texte ne cesse pas un instant de se demander ce qui, du vivant, a une fin et ce qui, en lui, est sans fin bien que la vie, elle, finisse. Mais est-ce, après tout, si étonnant que la question des fins de la psychanalyse recoupe celle des fins du vivant? La psychanalyse ne vise-t-elle pas à étudier ce qu'il en est des processus de la vie psychique et ne tente-t-elle pas, par la cure, de réduire, autant qu'il se peut, ce qui vient faire obstacle au psychique dans sa fonction de rendre vivable la vie?

Peut-on raccourcir la durée de la cure psychanalytique? L’espoir “d'éliminer la névrose entière" est-il "réaliste”? Est-il "possible de résoudre un conflit pulsionnel définitivement et pour toujours"? Peut-on "vacciner" un homme contre toutes les possibilités de conflit pulsionnel ultérieur? Peut-on traiter un conflit "qui ne se trahit actuellement par aucun indice”? Tel est l'ensemble de questions qui fonde le socle de la réflexion dans ce texte.

3. Ce terme est traditionnellement rendu en français par "annulation rétroactive”, la traduction par "rendre non advenu" que propose l'équipe de traduction des Euvres complètes, nous paraît plus rigoureuse. 
La légitimité des efforts pour raccourcir la durée des cures est reconnue comme un objectif "sensé et approprié", mais est souligné que, dans ce "désir thérapeutique”, peut aussi agir insidieusement "un reste de ce mépris impatient avec lequel une période antérieure de la médecine avait considéré les névroses “comme les suites superfétatoires de dommages invisibles. Dès lors qu'on était obligé de s'en occuper, on voulait du moins en finir avec elles le plus tôt possible” (Freud, 1937, p. 231). Rank fit une tentative, dans ce sens, particulièrement énergique. Elle se fondait sur la supposition que la "fixation originaire" à la "mère" pouvait être défaite et que sa persistance en tant que "refoulement originaire" pouvait cesser. Freud était moins optimiste quant au pouvoir de la cure, car il prenait en considération la constitution même de l'humain: ce point où l'on touche à ce qui le constitue certes singulièrement, mais dans les limites des compossibles propres à l'espèce. La cure psychanalytique ne saurait donner à un individu plus de pouvoir que la vie n'en donne à l'espèce humaine et elle ne peut réussir là où la vie ne saurait qu’échouer: "La psychanalyse ne réalise chez le névrosé rien d'autre que ce à quoi réussit l'homme sain sans cette aide” (Freud, 1937, p. 240).

Quant à éliminer la névrose entièrement, d'après Freud, à entendre, dans "la langue courante entre analystes", ce qui est dit "à propos d'un frère humain reconnu dans son imperfection", on pourrait croire que cela est possible, qu'il existe bien évidemment "une fin naturelle de l'analyse”. N'est-il pas, en effet et très souvent, dit avec regret et comme excuse: "son analyse n'a pas été finie, ou il n’a pas été analysé jusqu’à la fin” (Freud, 1937, p. 234). Mais, une telle “fin naturelle" existe-t-elle, est-il "même possible de mener une analyse à une telle fin"? À quel moment peut-on dire d'une analyse qu'elle est terminée? Mise à part, la mordante ironie de Freud: “L'analyse est terminée quand l'analyste et le patient ne se rencontrent plus pour l'heure de travail analytique”, deux significations sont données à la fin de l'analyse. Selon la première, la cure est terminée dès lors que "le patient ne souffre plus de ses symptômes et [a] surmonté ses angoisses comme ses inhibitions" et que, d'après le travail fait, l'analyste peut penser que l'on n'a "pas à craindre la répétition des processus pathologiques en question". Quand un tel but n'est pas atteint, "on préfère parler d'une analyse incomplète plutôt que d'une analyse inachevée”.

L'autre signification donnée à la fin d'une analyse est beaucoup plus ambitieuse: on se demande "si l'influence exercée sur le patient a été poussée si loin qu'on ne peut attendre de la poursuite de l'analyse aucune modification ultérieure". On suppose, donc, que l'on pourrait "atteindre par l'analyse un niveau de normalité psychique absolue, auquel on serait aussi en droit de faire confiance quant à la faculté de se maintenir stable, comme si d'aventure on avait réussi à lever tous les refoulements survenus et à remplir toutes les lacunes du souvenir". Pour Freud, cela ne va pas de soi et il faut interroger “d'abord l'expérience pour 
savoir si une telle chose arrive et ensuite la théorie pour savoir si même c'est possible" (Freud, 1937, p. 235).

Dans le cours de cette interrogation, la part constitutionnelle s'avérera être l'incontournable limite de l'action de la cure. Cette position de Freud sera, pour certains, le signe de son inanalysé, des limites de son savoir et de sa technique, mais, pour nous, elle résonne d'avantage comme une position d'humilité éthique signant un renoncement à un fantasme de toute-puissance de l'analyste.

Les attentes des optimistes présupposent que l'on peut atteindre un niveau de normalité psychique absolue alors que, pour Freud: "toute personne normale n'est en fait que moyennement normale, son moi se rapproche de celui du psychotique dans telle ou telle partie, dans une plus ou moins grande mesure..." (Freud, 1937, p. 238-39, 250).

\section{Une aporie: concilier des inconciliables, éviter l'insupportable}

Nous nous sommes étonnés que Freud évoque “sa” négligence de la dimension économique. En effet, dès le début de son œuvre, l'appareil psychique qu'il construit est destiné à maîtriser les excitations, à lier les quantités d'énergie en les transformant en qualité psychique. Dès L'interprétation des rêves, sa fonction est de maintenir le plus bas possible l'énergie interne de l'organisme et d'assurer sa stabilité (Freud, 1900, p. 456). “L’invention” de cet appareil surgit comme nécessité vitale face à l'excitation: danger intérieur devant lequel l'humain ne peut pas fuir. Il est la mesure et la trace de l'exigence de travail imposée par la pulsion au psychique au moment même de sa constitution en tant qu'appareil : dans un seul acte, la pulsion serait limitée, liée, endiguée dans un représentantreprésentation et se constituerait l'inconscient. L'organisme ne peut supporter le déplaisir que jusqu'à un certain seuil: au-delà la douleur surgit comme risque de destruction. La principale tâche de l'appareil est d'assurer son fonctionnement selon le principe de plaisir, cela n'est possible que si le débordement par l'angoisse automatique peut être anticipé et relativement évité.

Au sein de cet appareil, la tâche du moi est d'être l'intermédiaire, au service du principe de plaisir, entre le ça et le monde extérieur, de protéger celui-là de celui-ci tout en limitant autant qu'il se peut les exigences pulsionnelles du ça pour éviter le surgissement d'un excès de déplaisir. En un mot, le moi est tenu à une aporie: concilier les inconciliables afin de nous les rendre supportable en créant, dans l'appareil, un état de stabilité le plus permanent et le plus durable possible. Il y parvient en partie grâce à ses mécanismes de défense dont l'un des effets est la falsification de notre perception intérieure par une méconnaissance des rapports 
fondamentaux des forces qui animent l'appareil. Tout à son entreprise de maîtrise, le moi ne peut que négliger le point de vue économique puisque l’une de ses tâches serait de parvenir à faire des exigences pulsionnelles des quantités négligeables. La négligence dont parlait Freud serait constitutive et se révèlerait quand se fait entendre à nouveau la revendication pulsionnelle qui se manifeste alors bien plus “indomptée” que nous ne l'avions cru.

Les chances de la thérapie analytique tiennent à trois facteurs: l'influence des traumatismes, la force constitutionnelle des pulsions et la modification du moi. L'étiologie des névroses, toujours d'origine mixte, met en lumière l'action conjointe de deux de ces facteurs: le constitutionnel et l'accidentel ou traumatique. Le premier a trait à la force pulsionnelle qui s’avére "réfractaire au domptage par le moi”, le second dépend de l'action des traumatismes précoces dont le moi immature n'a pu se rendre maître, de leur action résultent des modifications du moi. Pour Freud, l'étiologie, où prédomine le facteur traumatique, "offre à l'analyse l'occasion de loin la plus favorable". Dans ce cas seulement, elle réussit ce dont elle capable: "substituer, grâce au renforcement du moi, une résolution correcte à la décision inadéquate remontant à l’âge précoce”. Cela se traduit par l'élimination du trouble névrotique qui ne fait plus retour et auquel aucun autre ne se substitue. C'est, dans ce cas, que l'on peut parler d'analyse définitivement terminée qui n'a plus besoin d'être poursuivie. Mais, même si le patient ne produit plus, par la suite, de symptômes l'obligeant à recourir de nouveau à l'analyse, cela ne pourra pourtant pas être attribué de manière certaine à l'analyse. On ne saura jamais dire ce qui, dans cette "immunité", relève de l'analyse ou des “faveurs du destin” qui lui auront épargné le poids de trop fortes épreuves.

Nous ferons une remarque: l'étiologie traumatique est certes la mieux indiquée pour la cure, mais cette analyse que l'on pourrait considérer comme définitivement terminée n'en demeure pas moins un cas d'école, une fiction. Car, pour un tel résultat, il faudrait qu'existe une névrose d'étiologie purement traumatique ou, pour le moins, une névrose dont la part constitutionnelle ne compterait pour ainsi dire pas. Or, toute névrose a une origine mixte, la limite de cette part indépassable parce qu'indomptable sera, donc, rencontrée dans toute cure. Et justement, quand dans l'étiologie prédomine ce facteur ainsi qu'une trop grande restriction de la force du moi, les conditions pour la psychanalyse sont défavorables. Face à la force du pulsionnel, dans les tous débuts, le moi se trouve démuni $\left(\right.$ hilflos $\left.{ }^{4}\right)$. Du secours apporté par autrui viendront les moyens psychiques

4. Nous sommes ici renvoyé à l'Hilflosigkeit, à l'état de détresse, ce moment initial dans lequel advient le petit humain, prototype de la situation traumatique. 
pour faire partiellement face aux exigences de la pulsion. Il semble que, pour Freud si, dès le début, la force du moi s’est montrée trop faible laissant la part belle au pulsionnel, la partie soit jouée et le pouvoir de l'analyse devenant vain: tout dépend, dès l'origine, et pour la suite, du "rapport de forces relatif des instances qui luttent entre elles” (Freud, 1937, p. 250).

Au moment où est abordée la question de savoir si un conflit de la pulsion avec le moi peut être liquidé définitivement, une précision, qui a des conséquences techniques, est apportée: "pour si décisif que puisse être au tout début le facteur constitutionnel, il n'en demeure pas moins pensable qu'un renforcement pulsionnel survenant plus tard dans la vie puisse manifester les mêmes effets. La formule serait donc à modifier: force pulsionnelle actuelle au lieu de constitutionnelle” (Freud, 1937, p. 239).

\section{Quand le moi cède... la force actuelle du constitutionnel...}

Certes, il se peut qu'aux tout premiers temps de la vie, par le refoulement originaire, aient pu se construire "des digues qui ne cèdent pas si facilement à la marée montante de l'accroissement pulsionnel” (Freud, 1937, p. 242). Mais si, cette force d'action constante peut être inhibée par l'action du moi et ne se manifester que, de manière supportable, sans trop entraver son fonctionnement, sa poussée n'en reste pas moins constante et toujours agissante. Pour qu'elle soit contenue, est nécessaire le déploiement d'un contre-investissement dont la force doit rester constante sous peine de voir le moi submergé par le pulsionnel. Mais, même quant il en est ainsi, l'équilibre atteint n'est que relatif et momentané. Car, rien ne nous protège des aléas de la vie et des effets du temps sur l'appareil psychique. Non seulement, un renforcement de la pulsion peut toujours survenir, mais, surtout, il se produira, avec le temps, immanquablement: toute son actualité originaire est alors redonnée, au présent, à la revendication pulsionnelle. Le moi ne dispose en effet d'aucun moyen pour faire disparaître définitivement la revendication pulsionnelle. Constitutionnellement, la force de la pulsion ne s'avère "domptable" que dans une certaine mesure et de manière non durable: à plus d'une occasion, le dompteur se découvre asservi par ce qu’il croyait avoir dompté.

La pulsion se voit renforcée de l'extérieur de l'appareil psychique: soit en raison des modifications physiologiques survenant au cours du développement (puberté, ménopause etc.), soit en raison d'influences accidentelles. Les deux cas produisent le même résultat: du corps ou de la réalité, provient un surcroît d'excitation qui accroit la force pulsionnelle, exigeant ainsi du psychique encore plus de travail pour maintenir ou rétablir dans l'appareil la stabilité menacée ou 
perdue. Ce qui est en jeu ce n'est pas l'âge, mais la nature de la pulsion, qui se manifeste bien alors comme un concept-limite entre le psychique et le somatique. Par elle, le psychique reste, quoique différencié, étroitement corrélé (zusammenhangen) au somatique. Dans ces situations, les symptômes ne s'avèrent être des formations de compromis que, pour un certain temps, au cours duquel le moi, se trompant lui-même sur la maîtrise acquise, croit pouvoir s'incorporer le symptôme en faisant de ce corps étranger une partie de son organisation. Mais le symptôme, tel le cheval de Troie, dans ses flancs, porte les forces que le moi tentait de dominer et qu'il croyait disparues. Avec le temps, s'estompe la dimension du compromis et le symptôme s'affirme de plus en plus comme formation substitutive de la motion refoulée, véritable représentant de celle-ci au sein même du moi qui se voit menacé de l'intérieur même de son organisation. Nous retrouvons la règle dégagée dans "l’homme aux rats": "ce qui doit être refoulé arrive avec le temps à pénétrer dans ce qui le refoule” (Freud, 1909, p. 246).

La négligence du point de vue économique est la méconnaissance de cette lutte fondamentalement inégale que le moi mène pour se rendre maître de la pulsion. Certes, le travail analytique peut être comparé, comme Freud l'a fait, au travail d'assèchement du Zuiderzee. Le moi parvient effectivement à édifier des digues contre la marée pulsionnelle, à gagner du terrain sur le pulsionnel. Mais, ce n'est là qu'un gain partiel, momentané. Car, la pulsion ne se laisse contraindre que pour partie et jusqu'à un certain point. Et, défiant le temps, inlassablement, elle use, de ses assauts répétés et constants, les digues que le moi, comme un Hollandais, défend dans le temps même où, tel un Troyen, il introduit, non pas un, mais plusieurs chevaux derrière ces mêmes digues.

L'un des résultats du travail psychique est de nous permettre de méconnaître le facteur quantitatif. La fonction de l'appareil est la transformation de la quantité en qualité. Il y parvient en donnant une représentation psychique à l'excitation pulsionnelle, moyen de lier celle-ci, premier pas, aussi, vers la maîtrise intellectuelle du monde. Sa tâche est de créer de l'ordre à partir du désordre pulsionnel. Qu'il y réussisse partiellement a pour effet de permettre d'ignorer momentanément la part laissée au chaos pulsionnel en nous, de méconnaître ce qui reste irréductiblement non lié. N'est retenu que le gain qualitatif tandis qu'est oublié ce qui, de la quantité, a échappé à la transposition psychique. L'appareil, tout à ses gains, méconnaît le résiduel des opérations qu'il accomplit. Celui-ci fonctionne pour l'appareil psychique comme "une immobilisation partielle en arrière” qui nous révèle que “tout progrès n’est jamais qu’à moitié aussi grand qu'il ne le paraît dès l'abord” (Freud, 1937, p. 243).

Ne plus négliger l'économique contraint à penser les limites de l'analyse. Celle-ci peut certes permettre de "parvenir plus d'une fois à éliminer l'influence 
du renforcement pulsionnel”, mais il est vraisemblable que son action se limite essentiellement "à élever la force de résistance des inhibitions [du moi] si bien que celles-ci seraient, après l'analyse, à la hauteur d'exigences bien plus fortes qu'avant l'analyse ou sans celle-ci” (Freud, 1937, p. 243).

\section{Viscosité de la libido... le poids de la fixation}

Par négligence du point de vue économique nous est désigné que, pour une part non négligeable, le pulsionnel s'avère non-maîtrisable par l'appareil psychique: apparaît ainsi sa dimension d'irréductibilité constitutionnelle. Une certaine quantité de pulsion demeure inapte à la conversion en qualité psychique et il subsiste toujours un certain désordre pulsionnel dont le pouvoir désorganisateur s'impose à l'appareil psychique. Le pulsionnel dont il est question ne serait-il pas celui que Freud vise sous le nom de pulsion de mort?

C’est, maintenant, par rapport à la manifestation d'une particulière "viscosité de la libido” que va resurgir la référence à l’âge. Dans certaines cures, les processus psychiques se déroulent bien plus lentement que, dans d'autres, les patients ne peuvent pas se résoudre à se détacher d'un objet et à déplacer les investissement sur un nouvel objet et cela sans que puisse être comprise les raisons d'une telle fidélité d'investissement (Freud, 1937, p. 256-257). Cette limitation de la plasticité de la libido, par sa forte fixation à un but, à un objet, à un stade libidinal, oppose une résistance à l'élaboration psychique. Des quantités d'énergie se voient ainsi immobilisées et rendues non disponibles pour le travail psychique, le processus est partiellement paralysé. Cette résistance semble “dépendre de rapports fondamentaux dans l'appareil psychique”. Dans d'autres cures, nous avons à faire au contraire à une trop grande plasticité de la libido qui rend les résultats analytiques trop précaires. Aucun investissement ne tient: "on a l'impression, non d'avoir travaillé dans l'argile, mais d'avoir écrit dans l'eau”. Et, enfin, dans d'autres cures, ce que nous rencontrons c'est l'épuisement de la plasticité: "tous les déroulements, relations et répartitions de forces s’avèrent inchangeables, fixés et figés”. S’il s’agissait de "personnes très âgées”, cet état des choses pourrait s'expliquer "par ce que l'on appelle la force de l'habitude, l'épuisement de la réceptivité, par une sorte d'entropie psychique”, mais, car il y a un mais, les patients évoqués sont juvéniles... Une fois de plus, ce n’est pas l'âge en tant que tel qui est la raison fondamentale de cet état des choses.

Les termes utilisés: viscosité, plasticité, fixation, entropie psychique, inertie psychique, ne sont plus d'un usage si fréquent et certains peuvent même paraître désuets. Ils sont associés à la notion de "constitutionnel” par laquelle est désigné 
ce qui en nous s'avère irréductible. Cette notion, prenant alors toute son épaisseur polysémique, ne renvoie plus seulement à l'ensemble des caractères congénitaux somatiques et psychologiques d'un individu, mais au moment constitutif de l'appareil psychique et de l'instauration des principes fondamentaux qui régissent le cours des événements psychiques.

La viscosité de la libido est en corrélation avec l'inscription dans la psyché du pulsionnel en tant que représentation (Freud, 1923, p. 62). La capacité de répondre totalement aux exigences de la libido semble faire défaut à l'homme. Immanquablement, il fait l'expérience d'une Versagung, de quelque chose qui, tant dans la réalité extérieure qu'intérieure, fait défaillance quant à la satisfaction de la libido. Celle-ci étant a priori "extraordinairement plastique", ${ }_{5}^{5}$ nombre de moyens détournés existent pour pallier partiellement cette défaillance (les tendance sexuelles peuvent s'interchanger, la satisfaction refusée à l'une peut s'accomplir au travers d'une autre). Cependant, la force pathogène de la Versagung reste importante car, pour s'opposer à elle, tous les hommes ne possèdent pas la même capacité de sublimation et peu d'entre eux s'avèrent capables de préserver la plasticité et la mobilité de la libido. Une tendance à la fixation de la libido sur des modes détournés de satisfaction se manifeste. Si le moi ne s'accommode pas de cette fixation et qu'il se dresse contre elle, alors "le moi éprouve un refoulement là où la libido a subi une fixation". Cette dernière est un des facteurs facilitant la régression: plus la fixation est forte, plus s'impose la tendance à fuir l'insatisfaction actuelle par la régression jusqu'aux éléments déjà fixés. Elle apparaît, dans ce contexte, comme le moyen de conserver toutes les positions libidinales antérieures dans lesquelles le principe de constance a pu être respecté. Par cette tendance à la fixation, se manifeste la peur du nouveau et la crainte de ne pas être capable, en cas d'abandon de la position déjà connue, de créer dans la suite du développement, de nouveaux états de compromis obéissant au principe de constance et permettant d'éviter le débordement par l'angoisse.

\section{Fixation et refoulement originaire, chemin d'exil}

La puissance d'action de la fixation est déterminée par son étroite corrélation avec le refoulement originaire:

5. Pour ce qui va suivre nous invitons le lecteur à se reporter au chapitre 22: "Points de vue du développement et de la régression. Etiologie” (Freud, 1915-1917, p. 319-336). 
Nous sommes fondés à admettre un refoulement originaire, une première phase du refoulement, qui consiste en ceci que le représentant psychique (représentant-représentation) de la pulsion se voit refuser ["versagt wird"] la prise en charge dans le conscient. Avec lui se produit une fixation; le représentant correspondant subsiste, à partir de là, inaltérable et la pulsion demeure liée à lui. (Freud, 1915, p. 48)

Dans un premier temps, se produit la Versagung dont la conséquence sera dans un second temps la fixation. La fixation de la représentation et la fixation de la pulsion à celle-ci se produisent dans un seul et même temps. Cette fixation originaire apparaît à Freud, qui s’oppose là à Rank, comme indépassable, inaltérable. La levée du refoulement originaire relève de l'impossible, mais, par contre, il s'avère avoir un redoutable pouvoir d'attraction sur tous les éléments à refouler. Nous retrouvons, à nouveau, l'idée d'un nombre premier indivisible.

L'appareil psychique n'a, avec le temps, pour unique destin que sa destruction par cela même dont il prend origine et dont il a tiré par transposition les matériaux qui le constituent. Pour l'appareil, la possibilité de durer ne dépendrait que de la capacité qu'il a, plus ou moins et, seulement, pour un certain temps, de fonctionner entre la menace de la dissipation de ses investissements libidinaux et la menace de rigidité cadavérique résultant d'un ordre répétitif parfaitement symétrique. L’homme se déploierait “entre le cristal et la fumée” (Atlan, 1979).

Deux dangers pèsent sur les processus psychiques: celui de l'inhibition, comme impossibilité d'aller de l'avant et celui de la régression, comme tendance à être toujours tirée vers l'arrière. Pour illustrer cela, Freud recourt à la métaphore de l'exil: celle d'un peuple qui, par nécessité vitale, doit s'exiler de sa terre d'origine vers de nouveaux territoires. La totalité du peuple ne parviendra, bien évidemment pas, au nouveau pays. Une partie disparaîtra en cours de route, certaines autres parties s'arrêteront en cours de route en se fixant à tel endroit. Le reste poursuivra son mouvement. Mais, en cas de danger ou de rencontre d'un ennemi, plus les fractions du peuple laissées en arrière seront importantes, moindre sera la force des fractions avancées et plus grandes les tendances à régresser pour se réfugier auprès des forces restées en arrière. La capacité de survie d'un peuple réside dans son aptitude à affronter l'exil.

Le fait que la pulsion soit, tout au long de l'entreprise freudienne, maintenue comme un concept-limite entre le psychisme et le somatique, nous autorise à poursuivre cette métaphore. La tâche de l'appareil psychique est de permettre le dégagement de l'emprise de la perception sensible par l'exil du pulsionnel vers sa représentation. À ce travail, le temps d'une vie suffira à peine et nous nous demanderons avec R. Char (1983): “sommes nous voués à n'être que des débuts de vérité?” (p. 220). Plus importante, sera la part du pulsionnel qui n’a pas quitté sa terre somatique d'origine et plus une pression disloquante s'exercera sur le 
psychique à partir de ce qui sera resté immobilisé sur le chemin de l'exil. Les fixations fonctionneront comme une force d'attraction affaiblissant le processus d'élaboration. Et chacun des retours en arrière, qui s'avéreront parfois nécessaires pour se ressourcer auprès des forces restées en arrière, sera aussi, au même temps, menace de renforcement de la fixation. Sur le chemin de l'exil, pèse une autre menace, celle du clivage - c'est à dire de se couper trop radicalement de ses origines, de les dénier: dans celui que je suis devenu ne s'entendrait plus d'où je viens et existerait le risque d'une isolation coupant la représentation de l'affect. À chaque âge de la vie, ce n'est pas le nombre des années qui importerait, mais le point où nous en sommes quant à notre exil.

C'est dans les instants fugaces de perception de ce facteur irréductible que l'appareil psychique s'évertue à négliger que nous prendrions conscience de notre âge. Nous ne serions pleinement inscrit dans la temporalité que, dans les moments où, le moi ne pourrait plus négliger le point de vue économique. Nous prendrions, alors, conscience de ce qui, de la pulsion, a encore à être exilé dans le psychique pour y donner lieu à figuration. Dans ces moments, nous aurions l'obscure perception de l'usure de l'appareil psychique et du moi - plus particulièrement, de son usure à vouloir maîtriser ce dont il n'est après tout qu'une précaire et fragile différenciation.

Pour finir, nous citerons Daniel Pennac citant, dans "La fée carabine”, son père qui disait: “vieillir, hélas, mais c’est le seul moyen que j’ai trouvé de ne pas mourir jeune” (Pennac, 1987).

\section{References}

Atlan, H. Entre le cristal et la fumée, essai sur l'organisation du vivant. Paris: Ed. du Seuil, 1979.

Char, R. (1943-1944). Feuillets d’Hypnos. In: Euvres complètes. Paris: Gallimard, 1983. (La pléiade).

Freud, S. (1900). L'interprétation des rêves. 4. ed. Tradução Meyerson. Paris: PUF, 1976. (1904). Die Freudsche psychoanalytische Methode. In: Studienausgabe, Ergänzungsband. S. Fischer Verlag, 1989.

(1904). De la méthode psychanalytique de Freud. In: La technique psychanalytique. 6. ed. Tradução Anne Berman. Paris: PUF, 1977.

Verlag, 1989.

(1905). Über Psychotherapie. In: Studienausgabe, Ergänzungsband. S. Fischer 
(1905). De la psychothérapie. In: La technique psychanalytique. 6. ed. Tradução Anne Berman. Paris: PUF, 1977.

(1909). Remarques sur un cas de névrose obsessionnelle (l’homme aux rats). In: Cinq psychanalyses. 8. ed. Tradução M. Bonaparte et R. M. Lœwsenstein. Paris: PUF, 1977.

(1914[1918]). Extrait de l'histoire d'une névrose infantile. (L'homme aux loups). In: Cinq psychanalyses. 8. ed. Tradução M. Bonaparte et R. M. Lœwsenstein. Paris: PUF, 1977.

(1914[1918]a). A partir de l'histoire d'une névrose infantile. Tradução J. Altounian, P. Cottet. In: Euvres complètes, psychanalyse. Paris: PUF, 1988. t. XIII.

(1915). Le refoulement. In: Métapsychologie. Tradução de J. Laplanche et J.-B. Pontalis. Paris: Gallimard, 1978.

(1915-1917). Introduction à la psychanalyse. Tradução S. Jankélévitch. Payot: Paris, 1978. (Petite Bibliothèque Payot).

(1923). Psychanalyse et Théorie de la libido. Tradução J. Altounian, A. Bourguigno, P. Coter, A. Rauzy. In: Résultats, idées, problèmes. PUF: Paris, 1985. v. II.

(1937). L'analyse avec fin et l'analyse sans fin. Tradução J. Altounian, A. Bourguignon, P. Cottet, A. Rauzy. In: Résultats, idées, problèmes. PUF: Paris, 1985. v. II. Laplanche, J. Terminologie raisonnée, article “terrasser”. In: Traduire Freud. Paris: PUF, 1989.

Pennac, D. La fée carabine. Paris: Gallimard, 1987.

Villa, F. La mort n'est pas la conséquence du vieillissement. Réflexions sur les effets du temps sur les processus psychiques. Topique, Paris, Éd. L'esprit du temps, n. 81, p. 169-195, 2002.

A propos de l'ordinaire et extraordinaire détermination humaine à rester en vie. Champ psychosomatique, Éd. L’esprit du temps, n. 35, p. 103-127, 2004.

La puissance de vieillir, "une façon de commencer à devenir anorganique”. Psychologie clinique et projective, v. 11, p. 289-305, 2005.

\section{Resumos}

É a idade uma contra-indicação para o tratamento psíquico? O autor mostra que, por trás do problema da idade, o que se deve levar em consideração é a dimensão econômica.

Palavras-chave: Econômica (dimensão), pulsão, tratamento psíquico, envelhecimento 
¿La edad es una contraindicación para el tratamiento psíquico? El autor muestra que, por detrás del problema de la edad, es la dimensión económica la que debe llevarse en cuenta.

Palabras claves: Económica (dimensión), envejecimiento, pulsión, tratamiento psíquico

Can age be a counter-indication for psychic treatment? The author shows that the economic dimension behind this question must be considered.

Key words: Economic (dimension), drive, psychic treatment, aging

\section{François Villa}

Psicanalista; mestre de Conferência em Ciências Clínicas Humanas, Universidade de Paris 7 - Denis Diderot (Paris, França).

26, rue de Paradis

75480 Paris - Cedex 10

França

e-mail: villa@paris7.jussieu.fr 